\title{
Medios de comunicación alternativos: rasgos, logros, obstáculos y retos
}

\section{Introducción}

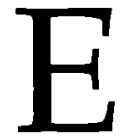

1 escenario político generado por los Acuerdos de Paz, ha influído positivamente en el ámbito de la comunicación y la expresión en general, así como en la orientación y modo de uso de los medios de comunicación en particular. Hoy día, por ejemplo, se ejercen con mayor garantía los derechos a la libre expresión y libre difusión de las ideas; aunque últimamente, preocupa a los medios informativos la regulación de la información propuesta en la reforma del sistema de administración de justicia (que pretende restringir la libertad de información, para salvaguardar el derecho de presunción de inocencia de las personas acusadas de un determinado delito), la cual ha sido calificada como "ley mordaza". Por otra parte, los formatos y contenidos que encontramos en un buen número de medios, son más profesionales y más pluralistas (a pesar de las tradicionales excepciones). No obstante lo anterior, el impacto del proceso democrático sobre la comunicación, la libre expresión y el uso de los medios, no debe limitarse a estos logros que, por muy positivos que hayan resultado, siguen siendo insuficientes para hablar de una comunicación realmente democrática.

El planteamiento que pretendemos sustentar en este artículo, es que una condición básica para avanzar hacia la democratización de la palabra, lo constituye la potenciación, consolidación y desarrollo de los medios de comunicación populares, educativos y participativos (medios alternativos). Del fortalecimiento de éstos depende, en buena medida, la vigencia de la democracia en este plano, por demás sustancial en la búsqueda de la democratización global de la sociedad.

Si el proceso democrático pasa -entre otras cosas- por el fomento y amplio desenvolvimiento de todas las corrientes de pensamiento, por el cultivo de la racionalidad histórica y por el fin de los dogmatismos y 
fanatismos impositivos. Si teóricamente hablando, los medios de comunicación deben formar parte del bien común, esto es, del conjunto de bienes naturales, económicos, sociales y culturales, a los que tienen acceso el mayor número de personas $y$, por tanto, deberían estar controlados democráticamente por toda la sociedad. En consecuencia, la puesta en práctica de estos presupuestos, reconocidos formalmente como esenciales a la democracia, nos llevan a plantear - allí donde hay una legítima necesidad- la promoción del mayor número de periódicos, revistas, radios, canales de televisión y videos que reflejen la gama más amplia posible de opiones e intereses de la sociedad.

Los intereses comerciales y estatales están suficientemente representados; no corren la misma suerte, los intereses de la sociedad civil. Los medios alternativos pueden constituirse en instrumentos claves, para que el sentir y las necesidades de los ciudadanos, formen parte de la conciencia colectiva y tengan incidencia en aquellos centros de decisión, sean institucionales, grupales o personales, que determinan cuestiones importantes de la marcha de la sociedad.

Tres son los puntos que configuran nuestro planteamiento: primero, dejaremos establecido en qué puede estar "lo alternativo" de los medios de comunicación llamados alternativos; segundo, haremos una descripción de la realidad actual de estos medios en nuestro país $y$, finalmente, analizaremos algunos retos o desafíos que enfrentan tales medios, de cara a fortalecer el proceso democrático.

\section{Caracterización de lo alternativo en los medios de comunicación}

Quizás sea lugar común afirmar que los medios de comunicación han cobrado un rol de primera importancia en estos tiempos de neoliberalismo y globalización. Sin embargo, creemos necesario recordarlo, no sólo porque hoy más que nunca son ellos quienes organizan gran parte de la vida social, sino también porque es justamente, en este marco y frente a ese rol, donde se configura la respuesta de la comunicación alternativa.

La centralidad de los medios deriva no sólo de su mayor consumo y expansión, sino a su vez, del hecho de ir ocupando lugares nuevos en la escena social y cumpliendo nuevos papeles. Enunciemos los rasgos más sobresalientes de este nuevo rol: los medios se han convertido en los legitimadores de hechos e ideas; fijan la agenda de lo que es o no es noticia, de lo que es tema de debate, de lo que es relevante; los medios masivos se han convertido en los principales espacios de representación e interección social,la escena política se construye principalmente en ellos ; en lo que respecta a la propiedad de los medios, ésta se ha constituido ( a nivel mundial, continental, regional y nacional) en un verdadero mono- 
polio que manejan industrias o consorcios de alta influencia económica y política; la extrema privatización que existe también en este campo, ha implicado una restricción significativa de la oferta de programas de servicio y desarrollo social; los medios estrictamente comerciales, promueven sólo un tipo de cultura: la del mercado neoliberal(la competencia y la rentabilidad son los referentes de la vida personal y social); en lo concerniente a las reglamentaciones legales de la comunicación y el uso de los medios, en la mayoría de nuestros países las leyes vigentes o la ausencia de las mismas, se convierten en serias limitaciones para el desarrollo de otros medios de comunicación con propuestas democráticas (ver ALER, 1994).

Es cabalmente en el marco de estas condiciones, donde se pretende reimpulsar una oferta comunicativa alternativa. Oferta que responda a las exigencias y retos de los nuevos tiempos, sin dejar de ser alternativa. Surgen entonces, dos preguntas inevitables: ¿ en qué consiste "lo aternativo" de la experiencia comunicativa y de dónde deriva su necesidad?

Ante todo hay que decir que lo alternativo ha resultado ser un concepto histórico, es decir, por referirse a realidades cambiantes que dependen de la situación estructural y coyuntural en la cual se da, ha ido cobrando un significado distinto, según el momento del proceso y según el contexto en el que surge. No es la misma comunicación alternativa la que pueda darse en un contexto de prohibición y negación total de los derechos ciudadanos, que en otro de aparente o real apertura democrática.

Por ejemplo, en aquellas etapas de nuestra historia de mayor cierre de los espacios de comunicación y de expresión, de mayor distorsión y ocultamiento de la verdad, de mayor persecución contra personas e instituciones, que pudieran representar un análisis objetivo y crítico de la realidad, así como identificación con los sectores populares; lo alternativo se definió por su oposición al orden vigente. El énfasis principal estaba en ser instancia crítica y desenmascaradora de la ideología e intereses predominantes. Como contraparte y para ocultar o negar lo que de legítimo había en este tipo de comunicación, los sustentadores del "orden" recurrieron a satanizar lo alternativo calificándolo de práctica ilegal, desestabilizadora y clandestina.

Por otro lado, en contextos de mayor apertura democrática, la comunicación alternativa ha logrado tornarse en principio de democratización no sólo de las comunicaciones, sino también de la sociedad como totalidad. Aquí el énfasis ha estado puesto en la liberalización de los flujos informativos (mayor pluralidad de fuentes), en la posibilidad de que todos los actores sociales ejerzan sus derechos a comunicarse y expresarse (irrestricto acceso a canales y espacios) $y$, en favorecer el desarrollo de una conciencia colectiva responsable, crítica y creadora. 
Lo anterior no significa que lo alternativo sea un concepto equívoco (tan flexible que pueda recibir toda clase de contenidos), al contrario, si bien su verdad se puede ir midiendo en sus resultados y, sus contenidos pueden ir cambiando según la situación en la que se plantea, su sentido esencial se mantiene. ¿En qué consiste este sentido esencial de la experiencia de comunicación alternativa? Señalemos y expliquemos algunos de sus rasgos principales:

(a) Lo alternativo parte de la convicción de que el hecho comunicativo, comienza con la iserción en la realidad de sus destinatarios, esto es, se sitúa en el mundo de aquella parte mayoritaria de la sociedad. El objetivo central de esta inserción no es sólo conocer los problemas, necesidades, intereses y esperanzas de esas mayorías; sino sobre todo, saber lo que tiene que comunicarse, cómo debe hacerse y qué lenguajes son necesarios para establecer una verdadera comunicación. Por tanto, frente a los medelos comunicativos, que más bien son impositivos, y que reducen a los destinatarios a ser consumidores potenciales o reales de cosas e ideas; lo alternativo reconoce, en los destinatarios, a verdaderos interlocutores cuya realidad y sentir orienta el horizonte de la acción comunicativa. Esos interlocutores están constituidos por lo que hoy se denomina sociedad civil, esto es, aquellos sectores de la sociedad que no pertenecen ni al estado ni al mercado. En tal condición estarían tanto el conjunto de los ciudadanos, como los sindicatos, universidades, asociaciones profesionales, de género, cooperativas y gremiales. La comunicación aternativa,entonces, asume como condición básica situarse en la realidad de la sociedad civil, para servirle mejor y para que ese servicio responda a los intereses del bien común.

b) Otro rasgo constitutivo, lo representa la decisión de posibilitar el poder de la palabra a aquellos sectores a los que históricamente se les ha marginado de la misma, sin que ello implique discriminación o exclusión de otros sectores. Frente a una práctica exclusiva y excluyente en el uso de los medios de comunicación, donde el ejercicio efectivo del derecho a la expresión ha sido un privilegio de pocos; lo alternativo propone democratizar la palabra. Por consiguiente, la comunicación alternativa es fundamentalmente participativa y lo es al menos por dos razones: primero, porque rompe el esquema unidireccional y elitista de la comunicación dominante (la difusión de mensajes verticalmente producidos es transformada por una comunicación entre iguales) $y$, segundo,porque busca favorecer la participación de la sociedad civil en el ámbito político, social, económico y cultural (el perceptor colectivo asume un papel protagónico en el proceso comunicativo). Aquí la participación no es un recurso más para asegurar altos niveles de audiencia y de circulación, ni una estrategia mercantil rentable; el propósito principal es más bien, democratizar el poder que da la palabra (ver Geerts,1995). 
Lo anterior supone la reorientación del uso de los medios de comunicación, para que éstos se conviertan en canales de expresión real de las personas y grupos excluidos, se conviertan en mediadores útiles entre los diferentes sectores de la sociedad civil y, entre ésta última y el estado; se conviertan, asimismo, en espacios de opinión que cuestione los discursos y prácticas antidemocráticas (ver Mata, 1995).

(c) Los medios de comunicación alternativos asumen también como tarea propia, el impulso y desarrollo de lo que podemos denominar la cultura del debate, esto es, el cultivo del razonamiento, la discusión, el análisis y la argumentación. Frente al fomento del individualismo, de la pasividad, el olvido y la indiferencia; lo alternativo propone el desarrollo y consolidación de una conciencia colectiva y personal con memoria histórica, capaz de generar una actitud responsable, crítica y creadora. En otras palabras, se plantea pasar del oscurantismo y la censura al ejercicio activo del saber pensar y discernir, conociendo las más variadas opiniones y posiciones socio-políticas.

(d) Lo alternativo se configura no sólo por el saber reponder a las exigencias de los interlocutores masivos, sino a su vez, por el saber hacer una comunicación a la altura profesional y técnica que demanda el mundo de hoy. Lo primero, ha conllevado la exigencia ética de servir a la verdad y a los sectores (campesinos, obreros, empleados, mujeres, jóvenes, niños, ancianos e indígenas) cuyos derechos humanos son violados por la pobreza extrema y por las distintas formas de discrinación social ; tanto la búsqueda y el anuncio de la verdad como la opción por mejorar la calidad de vida de las mayorías, representan los mayores y mejores logros de los medios de comunicación alternativos. Lo segundo (saber hacer comunicación), conlleva la necesidad de ser competentes y competitivos en un mundo altamente tecnificado; lo alternativo no tiene porque ser sinónimo de atraso o de mediocridad profesional, más bien debe estar abierta al desarrollo tecnológico de las comunicaciones, si quiere tener un mayor impacto en su entorno y si quiere ser competitiva técnicamente hablando. Por eso, la comunicación alternativa realiza en la actualidad serios esfuerzos, para que su producto sea de la mejor calidad. Sin olvidar, claro está, que el fin último de estos esfuerzos es servir a un tipo de comunicación, que pretende ser herramienta para el desarrollo humano (especialmente de los más pobres y desposeídos de la tierra), que busca promover la justicia, la paz y la solidaridad entre los pueblos. Frente a los que sólo buscan ser competitivos motivados por el éxito y el lucro; lo alternativo pretende una oferta comunicativa de la mejor calidad, al servicio de algo mucho más determinante que la mera rentabilidad económica: hablamos de la posibilidad de construirnos como personas y sociedades libres. 
No están aquí planteadas todas las caracteríticas de lo que es y ha sido la comunicación alternativa, pero los rasgos que hemos descrito son suficientes para comprender su necesidad y su orientación fundamental. Democratizar la palabra supone en esta opción comunicativa: participación de los ciudadanos, defensa del bien común, servicio a la verdad, mediación para resolver los problemas de la vida cotidiana, cultivo del debate responsable y pluralista, acceso tanto legal como real, de la sociedad civil, a la propiedad de los medios de comunicación, acceso a nuevas tecnologías y, garantía efectiva de recepción y expresión para los legítimos movimientos sociales, políticos y culturales.

\section{Situación actual de los medios de comunicación alternativos en El Salvador}

Un análisis aproximativo de la actual situación de dichos medios, sobre todo a partir de los Acuerdos de Paz, nos arroja las siguientes condiciones y tendencias:

(a) Es bastante constatable que el contexto socio-político originado por los Acuerdos, posibilita e impulsa un tipo de comunicación con menos censuras y con mayor pluralismo. Por ejemplo: los institutos políticos ejercen hoy, con más garantías que antes de los acuerdos, sus derechos a la organización, a la expresión y libre difusión de sus ideas; las organizaciones sociales encuentran nuevos espacios (aunque todavía restringidos), para indagar, cuestionar, criticar, denunciar y discrepar, sin que por eso se ponga en peligro inminente la vida de personas o instituciones; se han creado nuevos medios (especialmente escritos y radiofónicos) con un buen grado de pluralidad y profesionalismo, mientras que algunos de los antiguos, iniciaron cambios importantes en sus formatos y contenidos tanto de opinión como de información; se puso fin al control y censura que ejercían algunas instancias gubernamentales (SEN$\mathrm{CO}$, Fuerza Armada y Ministerio de Interior), sobre el quehacer informativo; asimismo, hay más diversidad en las fuentes consultadas y algunos temas y puntos de vista hasta hace poco ausentes, cobran hoy mayor presencia.

(b) Los aspectos arriba mencionados si bien son indicadores de que ha habido un importante movimiento en el quehacer de la comunicación social, éste todavía no es ni sufiente ni tan profundo como para hablar de una real democratización en este ámbito. El panorama sigue presentando serias limitaciones y graves vacíos(y no tanto ni principalmente por la amenaza de las regulaciones a la información). Enunciemos algunos de los puntos más sobresalientes en este sentido: primero,en términos puntuales el documento de los acuerdos circunscribió el tema de las comuni- 
caciones sociales a la garantía de la libre expresión y a los compromisos políticos para aprobar el funcionamiento legal de los medios de comunicación del FMLN, quedando ausente de una visión estructural sobre el uso y función democráticas de los medios; segundo, la comunicación social entendida como un derecho de la sociedad civil que implica: derecho a reunirse, a participar, a escuchar y ser escuchado, a ver y ser visto, a incidir en la configuración de la conciencia colectiva y en la toma de decisiones tanto políticas como económicas, todavía no presenta un grado aceptable de realización; tercero, el vigente monopolio de los medios no permite una presencia igualitaria a todos los puntos de vista, ni un suficiente acceso a los mismos por parte de la sociedad civil; cuarto, los medios de comunicación alternativos aunque han conquistado legítimos espacios, siguen limitados en su desarrollo debido, en unos casos, a que las autoridades respectivas o los vacíos legales, obstruyen su aspiración de funcionar en el marco de la legalidad y, en otros casos, por el ahogamiento económico de un mercado publicitario extremadamente monopolizado; quinto, la mayoría de medios de comunicación concentran su actividad en la venta agresiva de espacios publicitarios y en la legitimación de los hechos e ideas que aseguren la cultura neoliberal del mercado, subordinando la función social que deben tener los medios, esto es, potenciar la participación ciudadana, la identidad cultural, la formación de valores y el desarrollo democrático de la sociedad.

(c) Tanto el conjunto de estos logros como el de las limitaciones y vacíos, configuran, hoy día, el mapa de las comunicaciones sociales en nuestro país. Hagamos un breve rastreo poniendo especial énfasis en los medios de comunicación alternativos. Para ello, recurriremos a uno de los estudios más recientes titulado "Diagnóstico de la incidencia de la comunicación social en la reconciliación nacional de El Salvador" (ver UCA-CECI, 1995). Según esta investigación, los medios televisivos, radiales e impresos nacionales, se diferencian por su naturaleza y por su función; de tal manera, que podemos hablar de medios comerciales, estatales y alternativos.

(c.1) El sector comercial está compuesto por todos los comunicadores o medios de comunicación pertenecientes al sector privado. La generación y circulación de mensajes están, predominantemente, determinados por la venta de espacios publicitarios. Estos medios tienen una dinámica sustentada en dos ejes principales: por un lado, un alto nivel de competencia empresarial y por el otro, una fuerte vinculación con los sectores del poder político gubernamental. Esta dinámica junto con la alta concentración de propiedad, les posibilita: incidir de manera preponderante en el flujo comunicativo del país, una potente capacidad de difusión, desarrollar políticas comunicativas en bloque, tener control casi irrestricto sobre 
los contenidos de los mensajes, tener una posición ventajosa en la compra de programas y en el otorgamiento de exclusivas de difusión, estar a la vanguardia de las nuevas tecnologías $y$, tener control sobre el mercado publicitario a tal grado, que fácilmente puede cerrar espacios a la posible incursión de otros medios que operan fuera de su círculo. En términos cuantitativos este sector concentra por lo menos 10 televisoras, 15 productoras audiovisuales, 135 radioemisoras y 8 periódicos (entre tabloides y revistas).

(c.2) El sector estatal comprende a aquellos medios y comunicadores provenientes de instancias gubernamentales, instituciones autónomas o de la fuerza armada. Por estar fuera del mercado publicitario y por depender de un presupuesto estatal, podría esperarse que -en un contexto de paz- designaran sus recursos (2 radioemisoras, 2 televisoras, por lo menos 12 productoras de video, el diario oficial y numerosas revistas y folletos) de cara a cumplir con una genuina función social, es decir, orientar su peso comunicativo al servicio del bien común. Sin embargo, lo que realmente ocurre, es que en dichos medios pesa más el carácter oficioso (ser voceros del estado), y menos el derecho que tiene la sociedad de informarse y formarse en la verdad, la libertad, la justicia y la solidaridad.

(c.3) El sector alternativo está constituido por aquellos medios de comunicación vinculados directamente con instancias de la sociedad civil, tales como: universidades, organismos no gubernamentales, cooperativas y organizaciones comunales. Lo conforman cuando menos 25 instituciones que producen y/o difunden videos educativos o promocionales, 17 radioemisoras de carácter participativas o comunitarias y, un buen número de medios impresos (especialmente revistas y tabloides de circulación diaria o semanal).

Ahondemos un poco más sobre el estado actual de este sector, específicamente analicemos - tomados en su conjunto- sus principales logros, obstáculos y retos.

\section{(c.3.1) Logros de los medios alternativos}

Primero, hay que mencionar su proliferación -de hecho o de derecho- y gracias a los acuerdos de paz y a la iniciativa de diferentes instituciones de la sociedad civil, que reconocen el potencial de los medios de comunicación para consolidar la democracia, el pluralismo político e ideológico, el fomento de la identidad cultural y los valores humanos; en los últimos tres años se han multiplicado distintos esfuerzos que propulsionan un tipo de comunicación realmente democrática. En los medios impresos, por ejemplo, aparecieron 9 periódicos más: 4 semanarios, 3 quince- 
nales y 2 mensuales. A nivel de la producción y difusión videográfica, un buen número de organizaciones no gubernamentales y comunidades hacen uso del video ya sea para la búsqueda de ayuda finaciera o con propósitos educativos. El caso más llamativo lo constituye, sin duda, la proliferación de radios comunitarias. En la actualidad existen por lo menos doce radios de este tipo a lo largo y ancho de la geografía nacional (momentáneamente cerradas y silenciadas por orden de ANTEL). Todas ellas transmiten en baja potencia (la mayoría no pasa de 20 vatios), su cobertura es básicamente local o regional y, están ubicadas en las zonas más alejadas de la urbe.

Segundo, considerando el trabajo que estos medios realizan en su conjunto, podemos decir que se han convertido en nuevos espacios de comunicación de la sociedad civil. Sus objetivos y su práctica comunicativa apuntan hacia ese cometido. En lo que corresponde al quehacer videográfico-televisivo éste está orientado, por una parte, a acompañar el gestionamiento de ayuda financiera para proyectos de desarrollo social $y$, por otra parte, a la capacitación de los miembros de sus instituciones o comunidades, donde se hace un uso pedagógico de los videos de producción propia o ajenos. En momentos más coyunturales, sobre todo de abusos flagrantes a los derechos humanos, también se ha recurrido al video para ejercer una labor de crítica y de denuncia. Los nuevos medios impresos surgidos en el contexto de los acuerdos de paz (Tendencias, Opinión Ciudadana, La Voz y Primera Plana), han optado por un periodismo más independiente, más profesional y más pluralista, conscientes de que esta opción no sólo posibilita el hecho de que los medios se conviertan en instituciones democráticas; sino a su vez, en agentes democratizadores de la sociedad. El logro que aquí apuntamos es más notable, si analizamos el eje principal que configura el perfil de las radios alternativas: constituirse en medios de expresión de la sociedad civil y contribuir a mejorar la calidad de vida de la misma. Especial mención debemos hacer de las radios comunitarias, que se han convertido en primera fuente de información local y regional, en canales de convocatoria para actividades comunales, en espacios de encuentro y difusión de las expresiones culturales de la zona, en instrumentos de educación y orientación y, hasta en teléfeno público para la emergencia personal o colectiva.

Tercero, han logrado estructurar formatos pluralistas y participativos. La mayoría de estos medios han procurado estar abiertos a todos los sectores sin distinción ni discriminación. Asimismo, han posibilitado el cultivo de un tipo de comunicación más horizontal y, por ende, más participativo. Es cierto que este logro, sobre todo después de los acuerdos, no ha sido exclusivo de los medios que aquí llamamos alternativos. Algunos medios comerciales también han incursionado en un quehacer 
periodístico e informativo más democrático. Sin embargo, lo que diferencia a uno de otro es la motivación central que determina esta opción: los medios alternativos lo hacen porque su vocación propia es democratizar la palabra; los medios comerciales, en cambio, lo hacen sobre todo porque se han dado cuenta que informar y promover el debate entre los actores sociales y políticos, asegura tanto altos niveles de audiencia como de circulación, ambas condiciones necesarias para hacer de esta ámbito un negocio rentable.

Cuarto, ante la desintegración social y la atomización de las acciones de la sociedad civil, como las que se viven en la actualidad, es encomiable el surgimiento de la Asociación de Radios y Programas Participativos de El Salvador -ARPAS-, que aglutina las experiencias y esfuerzos de la comunicación radiofónica alternativa. Abrir espacios de asociación pluralista donde se busca contribuir al fortalecemiento del proceso democrático; donde se impulsa un sistema de comunicación participativo, dialógico y crítico; donde se fomentan los valores de la cultura democrática $y$, donde permanentemente se desarrollan proyectos de capacitación radiofónica, son logros importantes.

Quinto, los medios alternativos -especialmente las radios- han asumido como una tarea fundamental la democratización de la comunicación. Aunque este propósito es más bien un reto que una realidad, los esfuerzos que actualmente se realizan por actualizar el marco jurídico de las comunicaciones, así como los pasos dados para la consecución de la legalidad de las radios comunitarias, son ciertamente avances nada despreciables. El diagnóstico sobre comunicación en El Salvador, citado antes, destaca graves limitaciones de la actual legislación en esta materia, entre ellas: su carácter obsoleto, disperso y excluyente. De ahí que el estudio recomienda la urgencia de un nuevo marco jurídico de las comunicaciones que, entre otras cosas, regule la propiedad y uso de los medios de forma tal, que los derechos a la expresión y a la comunicación sean realmente ejercidos por la sociedad civil y no sólo por sectores o grupos privilegiados. El hecho de que las radios alternativas asociadas en ARPAS hayan tomado la iniciativa, es otro buen fruto que merece registrarse y al que deberían unirse otras instancias interesadas en democratizar este ámbito tan fundamental.

\section{(c.3.2) Obstáculos de los medios alternativos}

Uno de los obstáculos más sensibles que tienen las nuevas propuestas de comunicación social masivas y, más directamente los medios alternativos, es la existencia de un marco jurídico limitador y discriminador en el ejercicio de los derechos a la expresión y a la comunicación a través de los medios. Formalmente hablando, la Constitución Política garantiza, en 
su artículo 6, estos derechos y, por otro lado, existen regulaciones de carácter oridinario tales como: una ley de imprenta (1950), la ratificación de Convención sobre el Derecho Internacional de Rectificación (1958), medidas para el correcto uso de las publicaciones de noticias transmitidas por las agencias informadoras, decreto relativo a publicaciones hechas en cualquier medio de difusión del pensamiento por personas jurídicas, disposiciones que regulan el uso de obras de naturaleza intelectual por medios de comunicación y la participación de artistas salvadoreños en espctáculos públicos, decreto de la Junta Revolucionaria de Gobierno sobre los campos pagados publicados en los medios (1979), reglamento para el establecimiemto y operación de radiodifusoras (emitido en 1942 y con reformas en 1963), reglamento para teatros, cines, radio-teatros, circos y de espectáculos públicos (1948), ley de Fomento y Protección de la Propiedad Intelectual (1994). Existen además otzas normativas surgidas a raíz de los acuerdos de paz: el Acuerdo de San José (1990), en sus numerales 5 y 6 establecen que ha de darse plena garantía a la libertad de organización, de expresión y de prensa, al derecho de respuesta y al ejercicio del periodismo; el anexo F de los Acuerdos de Chapultuepec, exhorta a usar los medios de comunicación en favor de la reconciliación, mediante campañas nacionales de publicidad y abstenerse de toda propaganda o política informativa incongruente con los Acuerdos o con el proceso de distensión y de reconciliación; la propaganda y el proselitismo de los partidos políticos, deberán estar regulados sobre la base de la equidad y el respeto a la salud mental de los electores (Código Electoral, artículos 228 y 229).

No obstante todo lo anterior, el conjunto de estos decretos, disposiciones y reglamentos se caracterizan por su atomización, disparidad y, en la mayoría de los casos, por su falta de idoneidad y actualización frente a la realidad que pretende normar. Traemos a cuenta el caso que más conocemos: la situación que nosotros calificamos como de "ilegalidad forzada" en perjuicio de las radios comunitarias. En efecto, desde abril de 1995, la Administración Nacional de Telecomunicaciones (ANTEL), comenzó una campaña de cierre contra dichas radios, luego de negarles la autorización de frecuencia solicitada por éstas. La orden de cierre fue dirigida prácticamente a todas las radios comunitarias: radio Cooperativa (Santa Elena-Usulután), radio Victoria (Cabañas), radio Suchitlán (Cuscatlán), radio Ulúa (Cacaopera-Morazán), radio Excel (Saragoza-La Libertad), Teo-Radio (Teotepeque-La Libertad), radio Sumpul (Guarjila-Chalatenango), radio Izcanal (Nueva Granada-Usulután), radio Segundo Montes (Ciudad Segundo Montes-Morazán), radio Nejapa (Nejapa), radio Tecoluca (San Vicente) y radio Presencia (Santa Rosa de Lima-La Unión). Todas estas radios presentan características sumamente peculiares: transmiten en baja potencia, son básicamente rurales, los dueños o adminis- 
tradores son las mismas comunidades, gremios campesinos o alguna ONG de promoción social, su perfil radiofónico es educativo y participativo, la mayoría de ellas cuentan con el apoyo tanto de autoridades locales como de organismos de prestigio internacional (UNICEF, CREA INTERNACIONAL, La Asociación Mundial de Comunicación Cristiana - WACC, ALER y AMARC) y, finalmente, todas ellas surgen en el contexto de transición democrática.

No obstante, ANTEL en respuesta a las presiones de la Asociación Salvadoreña de Radiodifusores (ASDER) —que demandaba disponer las medidas encaminadas a poner término inmediato a dichas emisoras, por no estar autorizadas y hacer un uso indebido del espectro radioeléctrico (ver AIR,1995) - prohibió terminantemente el funcionamiento de dichas radios, alegando violación a la Ley de los servicios de Telecomunicaciones (ver ANTEL,1995) en sus artículos 7, 13, 25 y 26 (en ellos se estipula que la instalación y operación de una radio tendrá que estar autorizada por ANTEL, so pena de sancionar con multas a la persona que introduz$\mathrm{ca}$, instale $\mathrm{u}$ opere clandestinamente sistemas de telecomunicaciones). La prohibición se consumó con el cierre y confiscación de los equipos de transmisión de por lo menos 10 estaciones comunitarias. Medida ordenada por el presidente de ANTEL y ejecutada por la PNC el 4 de diciembre de 1995.

Las radios comunitarias en mención, aceptan que han estado transmitiendo sin tener el permiso de funcionamiento. Sin embargo, no ha sido éste su propósito: muchos son y han sido los esfuerzos realizados por estas radios para operar dentro de la ley. Pero, se han enfrentado con dos muros difíciles de derribar: en primer lugar,una ley que no se actualiza desde 1963 y que limita el uso de la radio a la esfera estatal y comercial, en menoscabo de las experiencias comunicativas que podrían estar al servicio de la cultura y del desarrollo social; en segundo lugar, la ausencia de voluntad política de las autoridades respectivas y de los sectores con mayor poder político y económico, que recurren a la ley no para hacer valer el derecho de acceso a los medios de comunicación, que tienen el ciudadano y la sociedad civil, sino para justificar lo contrario, es decir, la exclusión de éstos últimos.

Por comisión u omisión, tanto el carácter disperso y obsoleto de la ley como la falta de voluntad política mostrada, se han convertido por un lado, en eficientes instrumentos legales para mantener un uso monopolizado de los medios de comunicación y, por otra parte, en mecanismos excluyentes de los medios no estrictamente comerciales o estatales, así como de aquellos que, pretendiendo isertarse en el mercado de la comunicación predominante, no tienen posibilidad de sobrevivencia porque son 
ajenos a los grupos de mayor influencia económica, política e ideológica. Por eso consideramos, que las radios comunitarias se encuentran en una situación de "ilegalidad forzada" o, en todo caso, de "alegalidad" por cuanto no existe una ley que norme su origen, su naturaleza y su funcionamiento. En este campo, como en tantos otros, se hace necesario posibilitar una ley actual, justa y democrática.

Superar este desfase legal, que obviamente no sólo afecta a las radios comunitarias, sino a la misma necesidad de democratizar la comunicación, demanda entre otras cosas: la realización de un estudio de Derecho comparado en materia de libertad de expresión y de regulación de medios, corregir la dispersión legal para responder a la nueva realidad social y tecnológica que afecta a las comunicaciones; regular la práctica comunicativa comercial, de tal forma que no derive en monopolios, reorientar el uso de los medios de comunicación del Estado (mayor independencia del partido en el gobierno y mayor apertura a la sociedad civil), crear una instancia de carácter pluralista e interdisciplinaria, responsable del seguimiento de las leyes en materia de comunicación y de la práctica comunicativa del país (ver UCA-CECI, 1995). Todo ello, con el propósito de reactualizar y operativizar el espíritu de lo que la Constitución Política de El Salvador y la declaración universal de los Derechos Humanos, estipula en este campo, es decir: que el derecho a la libre expresión y comunicación es, ante todo, un derecho de la sociedad civil en su totalidad y no sólo ni exclusivamente de una de sus partes. A la sociedad política (estado, partidos políticos y afines) corresponde garantizar el ejercicio efectivo de estos derechos propios de la ciudadanía.

Otro de los obstáculos más serios que enfrentan los medios alternativos, es la existencia de una estructura de propiedad de los medios de comunicación monopólica. En efecto, hay una concentración de la propiedad de dichos medios en manos del gran capital, cuya omnipresencia en la mayoría de medios, da lugar a monopolios y círculos empresariales cerrados. Estas élites de poder, establecen las reglas del mercado de las comunicaciones e imponen sus intereses cuando éstos se ven limitados por competencias ajenas a su círculo o por normas legales contraproducentes a sus objetivos de rentabilidad (recordemos las protestas viscerales hechas por la Asociación Salvadoreña de Medios Publicitarios -ASMP - y la Asociación Salvadoreña de Radiodifusores - ASDER - a raíz de las regulaciones a las campañas electorales que establece el Código Electoral vigente).

Tal estructura centralizada y excluyente (fusión del gran capital, corporación de medios de comunicación comerciales y asociaciones de publicidad), no sólo no permite el desarrollo de los medios alternativos, sino incluso de aquellos medios con fines también comerciales, pero sin 
pertenencia al capital más grande y fuerte. Esta situación, en el caso de los medios objeto de nuestro análisis, genera un boicoteo político y económico, que no permite que los medios alternativos se desarrollen y se conviertan en instancias solventes económicamente hablando.

El acceso a la mayoría de paquetes publicitarios, por ejemplo, es casi imposible para los medios alternativos (las relaciones de propiedad y/o de asociación entre capital, medios y agencias, son las que en definitiva determinan las asignaciones publicitarias). En consecuencia, dichos medios son excluídos del mercado, teniendo casi como única opción de sobrevivencia la cooperación internacional que, en la actualidad, es cada vez más limitada y, en el mejor de los casos, sólo posibilita un funcionamiento para períodos relativamente cortos. En buena medida, esto explica lo que ocurrió en el periódico semanal Primera Plana, que se vio obligado a suspender temporalmente la publicación, debido al agotamiento del esquema financiero de la empresa que conllevó a una severa crisis de liquidez.

\section{(c.3.3) Retos de la comunicación alternativa}

La experiencia pasada y presente nos ha enseñado que esta opción es más difícil, más cara y más riesgosa. Pero, con todo, también es más humanizadora, más humanizante y más democrática.

Más difícil, porque, por un lado, supone ir más allá del modelo comunicativo predominante que conocemos: el modelo comercial que limita y condiciona sus mensajes a la combinación publicidad-entretenimiento-información (con dominio de la publicidad) o, el modelo estatal cuya actividad comunicativa es más oficiosa de los órganos que representa. En ambos casos, los intereses y necesidades de la mayor parte de la colectividad están escasamente representados. Pero, por otro lado, es más difícil porque supone en nosotros un gran esfuerzo de creatividad y formación, en lo que respecta al ejercicio personal e institucional de lo que implica una comunicación realmente democrática. Sin olvidar lo difícil que es incursionar en el mercado de la comunicación masiva, cuando se ofrecen formatos y contenidos alternativos; lo que indica que nuestra sociedad todavía no termina por tocar fondo en su democratización, ni por superar los dogmatismos y pragmatismos (políticos y económicos), que muy poco o nada tienen en cuenta los derechos e intereses de la sociedad como totalidad.

Más cara, porque si queremos democratizar la palabra y fortalecer la voz de la sociedad civil, esto demandará el uso de múltiples recursos humanos y materiales. Es más fácil y más rentable, limitarnos a un tipo de quehacer comunicativo comercial o estatal. Pero si hay que ir donde está la 
gente, si hay que buscar su participación, si hay que posibilitar un mayor acceso a los espacios, si nuestra producción tiene que ser de la mejor calidad (en su forma y en su contenido); necesariamente tenemos que contar con suficientes recursos que concreticen tales propósitos o potenciar al máximo los recursos modestos con los que se cuenta. En cualquier caso, se requerirá de un buen esfuerzo humano y de optimización de los recursos materiales.

Más riesgosa, porque si apostamos y hacemos una comunicación que busque y difunda la verdad frente a la mentira, que promueva la vida frente a la cultura de la muerte, que opte por la justicia frente a la justicia, por la honestidad frente a la corrupción $y$, por el respeto a la dignidad humana frente a pragmatismos utilitaristas; es bastante probable que más temprano que tarde, puedan producirse conflictos con nosotros mismos, cuando no somos coherentes entre lo que decimos y hacemos $y$, con todos aquellos (personas e instituciones) que pactan o hacen la mentira, la injusticia y la muerte.

No obstante lo anterior, la comunicación alternativa bien llevada, resulta ser: humanizadora, humanizante y democrática. Todo ello en tanto que posibilita la palabra a la sociedad civil (particularmente aquella parte mayoritaria que vive injustamente desposída de sus derechos fundamentales); favorece un pluralismo donde realmente haya diversidad, responsabilidad y compromiso con el bien común; opta por una comunicación horizontal (parte y se nutre de la vida misma de la gente y tiene en cuenta a la persona en su integridad), promueve espacios para el fomento de una cultura democrática, basada en la participación, el diálogo, la búsqueda de la verdad, así como en el respeto y promoción de los derechos fundamenteales del ser humano y de la naturaleza; está comprometida con el desafío de construir una cultura de la democracia que eleve, tanto la calidad de vida de las mayorías como la calidad de la conciencia colectiva y personal.

\section{Referencias bibliográficas}

AIR (1995). Resulución de la comisión sobre el uso indebido del espectro radioeléctrico de El Salvador. XXV Asamblea General Ordinaria de la Asociación Internacional de Radios - AIR - Cartagena de Indias.

ALER (1994). Hacia un nuevo horizonte teórico. IX Asamblea General Ordinaria. Quito.

ANTEL (1995). Resulución a todas las radiodifusoras comunitaria emitida por la presidencia de la Administración Nacional de Telecomunicaciones. San Salvador. 
Geerts, Andrés (1995). La comunicación alternativa en América Latina: balance y retos. San Salvador. (Mimeo).

Mata, María Cristina (1995). Uso de las nuevas tecnologías en comunicación social. En ALERTA (publicación de ALER), marzo. Quito.

Universidad Centroamericana "José Simeón Cañas" -UCA- y Centro Canadiense de Estudio y Cooperación Internacional - CECI- (1995). Diagnóstico de la incidencia de la comunicación social en la reconciliación nacional de $\mathrm{El}$ Salvador. San Salvador. (Mimeo). 